\title{
Towards Long-Term Intracranial Pressure Monitoring Based on Implantable Wireless Microsystems and Wireless Sensor Networks
}

\author{
J. Fernandes, P. M. Mendes \\ MEMS-UMINHO. University of Minho \\ Guimarães, Portugal \\ paulo.mendes@dei.uminho.pt
}

\author{
Carlos Abreu \\ Instituto Politécnico de Viana do Castelo \\ Viana do Castelo, Portugal
}

\begin{abstract}
Ambient Assisted Living (AAL) aims to provide support to healthcare professionals making use of sensing, and information and communication technologies. Brain related information is becoming more and more relevant for many pathologies, but access to long-term information from brain to feed such AAL technologies is still giving the first steps. One main issue when recording signal from the brain is the available room for sensing device placement. Since available room is limited, battery-less solutions are welcome. Also, AAL solutions to be developed should consider not only the sensing device, but also the entire supporting framework. This paper introduces a solution for long-term monitoring of intracranial pressure using a wireless microdevice and a wireless sensor network. This work introduces a solution to achieve an enough miniaturized pressure sensor, powered by a wireless link, and analyzes the suitability of a wireless sensor network to support the data dissemination.
\end{abstract}

Keywords-Ambient Assisted Living; Neuronal Microsystem; Wireless Power; Biomedical Application; Wireless Sensor Network; Physiological Signal; QoS

\section{INTRODUCTION}

The brain is the most complex organ in the human body. There are many physiological parameters that are measured in the human brain, and some of them are directly affected by the presence of tumors, aneurisms, and other disorders.

The pressure inside the human skull is a constant parameter that should be maintained between certain limits (usually 515 $\mathrm{mm} \mathrm{Hg}$ ) [1]. This pressure is called intracranial pressure (ICP) and is measured in the cerebrospinal fluid (CSF). An increase of the volume in the human cranium raises the intracranial pressure, which can subsequently damage human brain cells [2]. This condition is known as intracranial hypertension.

Intracranial pressure increase can be caused by different factors, and could give us information about other underlying problems in the brain. For example, the appearance of brain tumors can cause a tissue volume raise, and therefore can increase the intracranial pressure. In others cases the increase could be related with other problems. For example, a patient that has acute liver failure (ALF), also has its intracranial pressure raised [3]. An heart failure and other blood pressure (BP) related problems can also raise the intracranial pressure

This work was supported by Portuguese Foundation for Science and Technology: FCT-PTDC/EEI-TEL/2881/2012, Programa Operacional Temático Fatores de Competitividade (COMPETE) and Fundo Comunitário Europeu FEDER.
[4] as indicated by the relationship between blood pressure and intracranial pressure, called cerebral perfusion pressure $(\mathrm{CPP}=$ BP - ICP) [1]. Intracranial hypertension could also be related with some diseases like meningitis.

High values of intracranial pressure can cause brain damages and can be fatal if they remain high for a long time. ICP is also related with strokes, and can increase their probability of occurrence. Another disorder that can arise with chronically elevated intracranial pressure is Idiopathic intracranial hypertension (IHH). This disorder can cause a papilledema, which may lead to a progressive optic atrophy and, ultimately, blindness [5].

Due to the described problems, continuous intracranial pressure monitoring is recommended, commonly to guide therapy and prevent irreversible damage and secondary neurological deterioration [2].

There are many ways to measure the intracranial pressure, which have different advantages and disadvantages [6]. The most common way, involves the insertion of a tiny catheter in the human cranium, into the brain tissue to reach the cerebrospinal fluid. This technique is easily done but involves drilling into the human skull and it only measures ICP while the catheter is inserted and correctly positioned. Depending on the catheter it can also measure the oxygen partial pressure and brain temperature. Other techniques bring other advantages as less invasiveness (doesn't need to reach the cerebrospinal fluid) but with a loss in precision [6]. One recent technique involving a small flexible tube that is inserted into the lumbar spine surpasses the disadvantage of drilling into the human skull and can reach the cerebrospinal fluid [2]. There are other techniques that measure the intracranial pressure in different locations and with different types of sensors. However, one of the biggest disadvantages of these techniques is that they measure the ICP only during the procedure. In order to overcome this problem it is necessary to develop a microsystem that can be implanted into the brain and that can constantly transmit the ICP value without being necessary to insert a catheter again [6]. The implantation of a microsystem could enable a long-term measurement of the ICP while maintaining a good precision. 
The arteries, as well the volumes to place the pressure sensor in the brain are in the millimeters range [8], making possible to implant microsystems if they fulfill such dimensions. Moreover, an intravenous (catheterization) approach through the brain arteries may be envisaged to implant the microsystem avoiding the drilling of the skull. Using a self-folding technique [7] it's possible to fabricate microsensors and integrate them on a sub-mm microsystem, together with processing and communication sub-systems. Such dimensions make the microsystem implantation possible, at a moderate cost.

Wireless implantable microsystems are expected to revolutionize the way we monitor a patient [9]. However, most of works only look at particular issues of such huge problem. In this paper, a broader approach is used to propose a solution for long-term monitoring of intracranial pressure. We propose a solution to fabricate the tiny implantable pressure sensor, we investigate the possibility to power that device using a wireless link, and we investigate the response of a wireless network to data from that sensor.

\section{SySTEM ARCHITECTURE}

The overall solution should guarantee the long-term acquisition of intracranial pressure, transmission of such data using a network, and make that data available for the users and caregivers. In order to achieve that, first the pressure must be recorded by the wireless implantable microdevice and sent outside the head. Once outside, the pressure data will be routed by a dedicated wireless device to a general wireless device. Fig. 1 shows the overall system architecture.

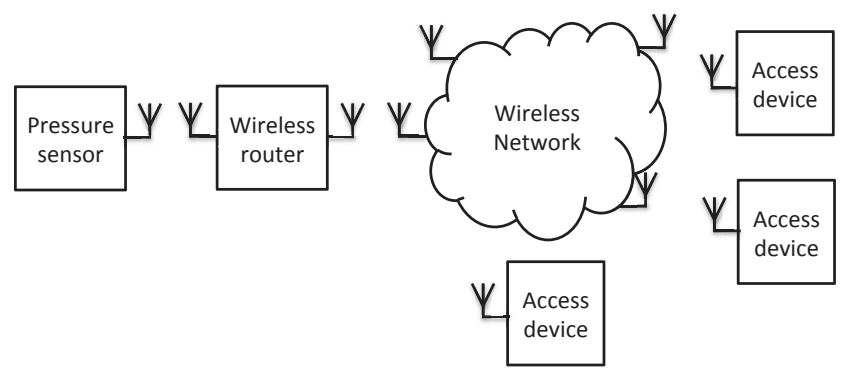

Fig. 1. Overall system architecture for intracranial pressure acquisition.

The pressure sensor is an implantable wireless microdevice, the wireless router is a device that will be placed close to the head and will act as an interface between the implanted pressure sensor and the outside world. The wireless router will make possible to obtain the pressure data and to wirelessly provide the required power to keep the pressure sensor running. The access devices should be any device, from smartphones to personal computers that will allow, and will provide access to the AAL system. The wireless network may be any network that allows the user to move freely everywhere, granting always the correct data delivery, with the required quality of service (QoS).

\section{A. Wireless Microdevice}

In this scenario, the most challenging sub-system is the wireless microdevice to measure the pressure. The device must be small enough (full system in the mm range [8]) to fit in the very small volume of interest and must provide a wireless link to send the measured data out, which requires the integration of an efficient antenna. Last but not least, pressure measurement in implantable devices is also a challenging issue $[9,10]$.

The solution proposed to obtain such small microdevice is to fabricate the device using CMOS technology, and integrate it with 3D antennas (fabricated using the self-folding technology) to allow the transmission and reception of radiofrequency (RF) fields. Fig. 2 shows an artistic view of the proposed wireless microsystem.

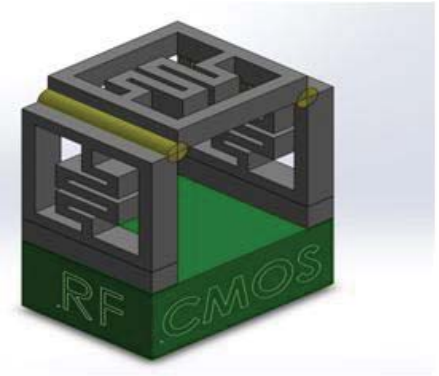

Fig. 2. Wireless microdevice obtained by integration of an RFCMOS device with an add-on module based on self-folding technology.

The CMOS technology allows to fully integrate the pressure sensor [11], the required RF circuitry to send data out and to receive the incoming RF signal that will be used to provide the power required for operation. The self-folding technology allows the integration of efficient 3D antennas.

\section{B. RF Link}

A fully functional pressure sensor can be operated using power levels from tents [12] to hundreds of microwatts [13]. Using more aggressive power saving solutions, it will be possible to reduce even further the power required for operation of the implantable pressure sensor. Since the full microsystem must be very small, it should not require a battery for operation to save space. In this way, it is required to use an energy harvesting mechanism. This paper proposal is to use an RF link to provide the required power for operation.

The RF link will be used for data transmission and to power up the device. The data transmission link should be as efficient as possible but is not very challenging since the wireless router has some room to accommodate a very sensitive receiver to detect the transmitted data. However, the RF link to power up the device should be as efficient as possible. Using the freespace path loss equation, it's possible to conclude that the power that will reach the implanted device depends on the antenna efficiency (the most challenging is the antenna in the implanted device, which in this proposal is 3D antenna obtained by self-folding to maximize the efficiency), and on the operating frequency. Increasing the operating frequency will have a positive impact in the antenna efficiency but the tissue loss increases. Decreasing the frequency will lead to less losses in the tissues but the antenna efficiency will degrade. In this way, a correct frequency must be selected in order to maximize the overall link efficiency.

Another aspect that should be taken into account is the standards available for wireless implantable devices and the 
standards available for wireless data transmission over wireless sensor networks (WSN). Regarding the WSN, the most common are Bluetooth, ZigBee, and WiFi, all available for operation in the $2.4 \mathrm{GHz}$ band. Regarding the implanted devices, we have the Medical Implant Communication Service (MICS), a standard that uses RF carriers in the $400 \mathrm{MHz}$ region, a frequency that will not suffer severe loss due to interaction with human tissues. However, since it requires large antennas, more recently the IEEE 802.15.6 [14, 15], also known as Medical Body Area Network (MBAN) was made available, allowing the use of a band close to the $2.4 \mathrm{GHz}$.

Considering that the preferred frequencies to transmit power into dispersive tissues are in the $1-10 \mathrm{GHz}$ range [16], the desired dimension for the implanted device (sub-mm), and the standard frequencies used in implanted devices, it was decided to implement an RF link in the 2-3 GHz range. Section on tests will show the measured attenuation for such a link.

\section{Supporting Wireless Network}

The main requirement for the wireless network in Fig. 1 is its ability to provide the required QoS. When considering biomedical applications, mostly, the QoS will be defined in terms of data delay (time from data acquisition to data deliver), and in terms of error rate (it may be bit error rate or packet reception rate - PRR). In same situations, the network persistence may also be an issue, however it may be disregarded once the data delay is guaranteed.

Sensor networks have been widely used in biomedical applications to transport highly demanding biosignals, as the electrocardiogram (ECG) or the electroencephalogram (EEG), where the data rate required is in the order of hundreds of kilobits per second per channel. The next section on tests will present data measured on a real scenario showing the possibility to run a wireless network with stable QoS for biomedical applications.

Depending on the pathology to be monitored, the required pressure data rate may change, but will be allays less demanding then ECG or EEG signals. The worst scenario would be to monitor the blood pressure at each hearth beat. However, it would be less demanding then the need to record the ECG waveform, or multiple EEG waveforms.

\section{Access Devices}

After being able to get the pressure signal outside the human head, it will be made available to different type of users through the wireless router and the wireless network. To assure data availability to the users of interest (the person being monitored and/or caregivers), an information and communication technology platform must ensure proper data access to different levels of permissions. Besides the framework to support all the interactions, it must work based on standard devices that everybody may have access, like smartphones or tablets.

To support the monitoring of different biosignals, we developed and tested a framework to allow the remote access and management of such signals [17]. The proposed solution was tested to display real time signals like ECG, based on medium range smartphones running the open source operating system Android.

\section{TESTS AND DisCUSSION}

The implementation of the proposed system faces two critical issues. One is the possibility to power-up the device using an RF link. The other is the possibility to send the data through a wireless network, being able to reach the destination for data processing and user feedback.

\section{A. Wireless Powering}

To test the attenuation expected by the RF link proposed by the technology of Fig. 2, the setup of Fig. 3 was used:

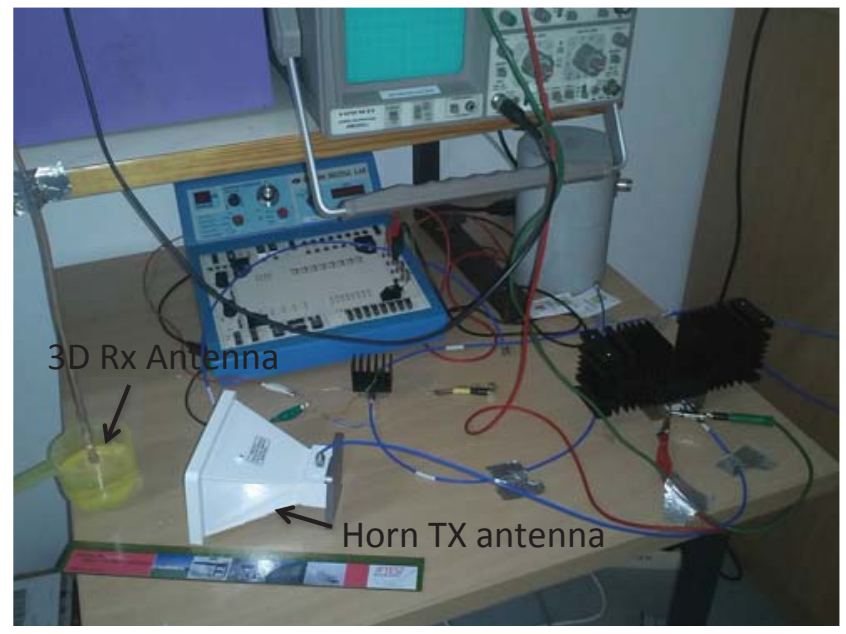

Fig. 3. Setup used to test the wireless link attenuation.

Fig. 3 shows the Q-Par wideband horn antenna used to feed the system, and the $3 \mathrm{D}$ receiving antenna immersed in a deionized water solution to mimic the presence of human tissue ( $5 \mathrm{~cm}$ around the antenna). The antennas were placed $10 \mathrm{~cm}(5$ $\mathrm{cm}$ solution plus $5 \mathrm{~cm}$ air) apart and a vector network analyser (E8357A PNA - $300 \mathrm{kHz}$ to $9 \mathrm{GHz}$ ) was used to measure the S21, giving an idea of path loss at different frequencies. Fig. 4 shows the measured results obtained.

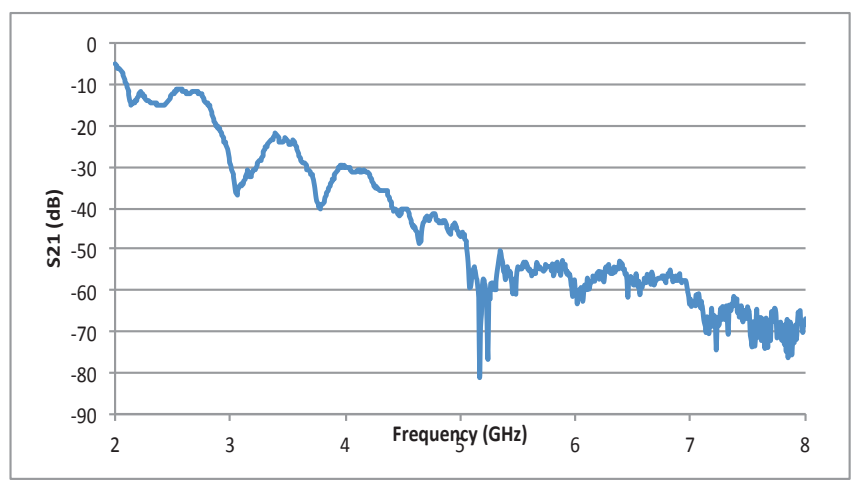

Fig. 4. Path loss of the RF link between an horn antenna and the 3D implanted antenna, considering $5 \mathrm{~cm}$ of solution and $5 \mathrm{~cm}$ of air.

From the previous figure, it's possible to observe that the path loss, despite being high, will make possible to feed the implanted device with powers in the range of tens of microwatts. 


\section{B. Network $Q o S$}

To understand if it would be possible to deploy the proposed monitoring solution, a wireless sensor network (based on IEEE 802.15.4) was deployed in a real hospital environment. Fig. 5 shows the deployment scenario.

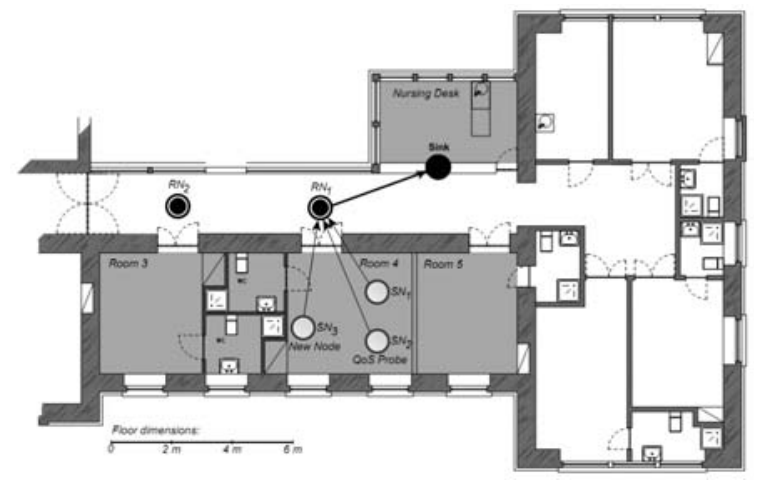

Fig. 5. Deployment scenario for a wireless sensor network to support the implantable pressure sensor in real hospital environment.

In this scenario we have three nodes in one room sending data trough the wireless network. Each node was programmed to send packets of 70 bytes each $500 \mathrm{~ms}$. To test network suitability, the QoS parameters used was the PRR. SN2 was used as a test node that would represent the implanted pressure traffic. To see the impact on the network, that node was turned $\mathrm{ON}$ and OFF, and we measured the PRR, as shown in Fig. 6.

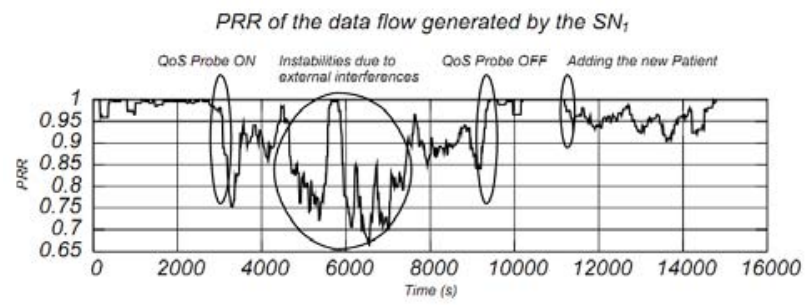

Fig. 6. Wireless network testing using PRR (Packet Reception Ratio) as the QoS measure to access the network quality.

Fig. 6 shows a severe degradation when severe interference was present. In this case several people were interfering with the propagating path.In what respect to the sole effect of data transmission, it can be observed the effect that turning ON (3000 s) and OFF (9500 s) the implanted sensor would have. It can be observed a degradation of the PRR, however this was a scenario were the data rate was selected to be very heavy for the network (each node send 70 bytes each $500 \mathrm{~ms}$ ). Even in this adverse scenario, with this experience, and others, we were able to conclude that such network would allow the implementation of the desired real-time monitoring of intracranial pressure using this standard network technology.

\section{CONCLUSIONS AND FUTURE WORK}

This work proposes a closed loop solution to supervise the intracranial pressure. The critical issues under analysis were device dimension, RF link for power and data transmission, and wireless network suitability for data transmission and automatic feedback to the user or to the caregivers. To obtain the desired device dimensions, a combination of RFCMOS standard technology with 3D fabrication technology was proposed and the required antenna was fabricated. The RF link was selected, implemented, and measured. It was verified that the resulting attenuation in the frequency range $2-3 \mathrm{GHz}$ will allow to power the microdevice with a wireless link. Finally, a wireless network was deployed in a real hospital environment to assess the possibility of using a standard WSN as a support for the pressure monitoring system. It was verified that, even with adverse conditions, the network was able to deliver the required data. This paper shows how to combine a set of technologies to implement and deploy a system to allow longterm monitoring of intracranial pressure.

\section{REFERENCES}

[1] L. Rangel-Castilla, L. Rangel-Castillo, S. Gopinath, C. S. Robertson, "Management of intracranial hypertension.," Neurologic clinics, vol. 26, no. 2, pp. 521-41, May 2008.

[2] V. Speck, D. Staykov, H. B. Huttner, R. Sauer, S. Schwab, and J. Bardutzky, "Lumbar catheter for monitoring of intracranial pressure in patients with post-hemorrhagic communicating hydrocephalus.," Neurocritical care, vol. 14, no. 2, pp. 208-15, Apr. 2011.

[3] J. Polson and W. M. Lee, "AASLD position paper: the management of acute liver failure.," Hepatology (Baltimore, Md.), vol. 41, no. 5, pp. 1179-97, May 2005.

[4] L. Friedfeld and A. M. Fishberg, "The Relation Of The Cerebrospinal And Venous Pressures In Heart Failure.," The Journal of clinical investigation, vol. 13, no. 3, pp. 495-501, May 1934.

[5] M. Wall, "Idiopathic intracranial hypertension.," Neurologic clinics, vol. 9, no. 1, pp. 73-95, Feb. 1991.

[6] J. Zhong, M. Dujovny, H. K. Park, E. Perez, A. R. Perlin, and F. G. Diaz, "Advances in ICP monitoring techniques.," Neurological research, vol. 25, no. 4, pp. 339-50, Jun. 2003.

[7] C. L. Randall, E. Gultepe, and D. H. Gracias, "Self-folding devices and materials for biomedical applications.," Trends in biotechnology, vol. 30, no. 3, pp. 138-46, Mar. 2012.

[8] S. Kamath, "Observations on the length and diameter of vessels forming the circle of Willis.," Journal of anatomy, 1981.

[9] I Kensall D. Wise, "Wireless Implantable Microsystems: Creating a Revolution in Health Care," Invited plenary address, Solid-State Sensor, Actuator, and Microsystems Workshop, Hilton Head, SC, June 2012

[10] Guangqiang Jiang, "Design challenges of implantable pressure monitoring system," Front. Neurosci., 26 February 2010

[11] Chia-Chu Chianga, Chou-Ching K. Linb, Ming-Shuang Ju, An implantable capacitive pressure sensor for biomedical applications, Sensors and Actuators A 134 (2007) 382-388

[12] Steve J. A. Majerus, Steven L. Garverick, Michael A. Suster, Paul C. Fletter, and Margot S. Damaser. 2012. Wireless, Ultra-Low-Power Implantable Sensor for Chronic Bladder Pressure Monitoring. J. Emerg. Technol. Comput. Syst. 8, 2, Article 11 (June 2012)

[13] Peng Cong, N. Chaimanonart, W. H. Ko, D. J. Young, "A wireless and batteryless $130 \mathrm{mg} 300 \mu \mathrm{W} 10 \mathrm{~b}$ implantable blood-pressure-sensing microsystem for real-time genetically engineered mice monitoring," Solid-State Circuits IEEE International Conference, 2009.

[14] Society, IEEE Computer, "IEEE 802.15.6 Wireless Body Area Networks," IEEE, 2012

[15] Fish's Regulatory \& Government Affairs Group, "Wireless Medical Technologies: Navigating Government Regulation in The New Medical Age," Terry G. Mahn, Chair, 2013.

[16] A.S.Y. Poon, S. O’Driscoll, and T.H. Meng, "Optimal frequency for wireless power transmission into dispersive tissue," IEEE Trans. Antennas and Propagation, 58(5):1739-1750, May 2010.

[17] J. Festa, C. Silva, P. M. Mendes, "Health Monitoring Platform for AAL Applications," Proc. BIODEVICES 2012, Vilamoura, Portugal, 01-04 February, 2012. 